Pacific Journal of Mathematics

SIMPLICITY OF PARTIAL AND SCHMIDT DIFFERENTIAL 


\title{
SIMPLICITY OF PARTIAL AND SCHMIDT DIFFERENTIAL OPERATOR RINGS
}

\author{
D. R. MALM
}

In this paper we develop necessary and sufficient conditions for certain differential operator rings to be simple. We do this for a ring with finitely many commuting derivations and for a ring with a commuting Schmidt higher derivation of finite length. Also we give a correspondence between finite sets of derivations and Schmidt higher derivations.

In this first section we deal with the simplicity of a differential operator ring over a ring. with finitely many commuting derivations. Many of the results of this section overlap those of others, among them Jordan [8], Hauger [5] and Voskoglou [11]. The second section deals with the construction of a Schmidt higher derivation from a set of derivations, and vice versa. The correspondence is the same as that of Heerema [6] but we give a different construction. The third section deals with simplicity of a differential operator ring over a ring with a Schmidt higher derivation. All rings are considered to be associative with unit. This research will form part of the author's Ph.D. dissertation at the University of Utah. The author wishes to thank K. R. Goodearl for his help and suggestions.

1. Let $\delta_{1}, \ldots, \delta_{k}$ be derivations on a ring $R$. A $\left(\delta_{1}, \ldots, \delta_{k}\right)$-ideal of $R$ is any ideal $I$ of $R$ such that $\delta_{i}(I) \subseteq I$ for all $i$. The ring $R$ is said to be $\left(\delta_{1}, \ldots, \delta_{k}\right)$-simple if $R$ is nonzero and the only $\left(\delta_{1}, \ldots, \delta_{k}\right)$-ideals of $R$ are 0 and $R$. The elements $r \in R$ such that $\delta_{i}(r)=0$ for all $i$ are called $\left(\delta_{1}, \ldots, \delta_{k}\right)$-constants and form a subring of $R$.

If $\delta_{1}, \ldots, \delta_{k}$ are commuting derivations on $R$, the formal linear differential operator ring

$$
T=R\left[\Theta_{1}, \ldots, \Theta_{k} ; \delta_{1}, \ldots, \delta_{k}\right]
$$

is the free left $R$-module generated by the symbols

$$
\Theta_{k}^{n(k)} \ldots \Theta_{1}^{n(1)}
$$


with each $n(i)$ a nonnegative integer and given a ring structure by the relations

$$
\begin{gathered}
\Theta_{i} \Theta_{j}=\Theta_{j} \Theta_{i} \\
\Theta_{i} r=r \Theta_{i}+\delta_{i}(r)
\end{gathered}
$$

for all $i, j$, all $r \in R$, together with the usual multiplication in $R$. It is convenient to think of the elements of $T$ as polynomials, with left coefficients in $R$, in the $\Theta$ 's. For a $k$-tuple $I=(i(k), \ldots, i(1))$, with each $i(j)$ a nonnegative integer, let

$$
|I|=i(k)+\cdots+i(1)
$$

and

$$
\Theta^{I}=\Theta_{k}^{i(k)} \ldots \Theta_{1}^{i(1)}
$$

and call $I$ the order of $\Theta^{I}$. Let $N=(n(k), \ldots, n(1))$ and $M=$ $(m(k), \ldots, m(1))$. We say the order of $\Theta^{N}$ is greater than that of $\Theta^{M}$ if $|N|>|M|$ or, if $|N|=|M|$, if there exists $j \in\{1, \ldots, k\}$ such that $n(i)=m(i)$ for $i>j$ and $n(j)>m(j)$. Otherwise the order of $\Theta^{N}$ is less than or equal to that of $\Theta^{M}$.

For nonzero $t \in T$, the leading coefficient of $t$ is the coefficient (in $R$ ) of the monomial of highest order in $t$ with nonzero coefficient. We will usually denote an element $t \in T$ by

$$
t=\sum \alpha_{I} \Theta^{I}
$$

with $\alpha_{I} \in R$. If $\alpha_{J}$ is the leading coefficient of $t$, the order of $t$ is said to be $J$. One should note that for nonzero $t, s \in T$, the leading coefficient of $t s$ is the product of the leading coefficients of $t$ and $s$ unless that product is zero. When $k=1$, the order will be denoted by a lower case letter.

LEMMA 1. Let $\delta_{1}, \ldots, \delta_{k}$ be commuting derivations on a ring $R$ and $F$ the subring of central $\left(\delta_{1}, \ldots, \delta_{k}\right)$-constants of $R$. If the differential operator ring

$$
T=R\left[\Theta_{1}, \ldots, \Theta_{k} ; \delta_{1}, \ldots, \delta_{k}\right]
$$

is simple then the center of $T$ is $F$ and $R$ is $\left(\delta_{1}, \ldots, \delta_{k}\right)$-simple.

Proof. Certainly $F$ is contained in the center of $T$ and any element of $R$ central in $T$ is in $F$. So it will be enough to show that the center of $T$ is contained in $R$ to show $F$ is the center of $T$.

If $A$ is a proper nonzero $\left(\delta_{1}, \ldots, \delta_{k}\right)$-ideal of $R$, then $A T$ is a proper nonzero ideal of $T$. Hence, $T$ simple implies $R$ is $\left(\delta_{1}, \ldots, \delta_{k}\right)$-simple. 
As $R$ is $\left(\delta_{1}, \ldots, \delta_{k}\right)$-simple, any nonzero central $\left(\delta_{1}, \ldots, \delta_{k}\right)$-constant is invertible and therefore $F$ is a field.

Suppose $t=\sum \alpha_{I} \Theta^{I} \neq 0$ is central in $T$ with leading coefficient $\alpha_{J}$ and order $J$. Since $\Theta_{i} t-t \Theta_{i}=0$ for all $i$ and $r t-t r=0$ for all $r \in R$, we get $\alpha_{J} \in F$. Since $T$ is simple, $t$ is invertible. If $|J|>0$, and the leading coefficient of $t^{-1}$ is $\beta_{N}$, then $\beta_{N} \alpha_{J}=0$ implying $\beta_{N}=0$, a contradiction. Therefore $|J|=0$ and $t \in R$.

We can think of the derivations $\delta_{1}, \ldots, \delta_{k}$ being defined on $T$ such that for $t \in T$

$$
\delta_{i}(t)=\Theta_{i} t-t \Theta_{i}
$$

Note that this implies $\delta_{i}\left(\Theta_{j}\right)=0$ for all $i, j$ and if $t=\sum \alpha_{I} \Theta^{I}$, then $\delta_{i}(t)=\sum \delta_{i}\left(\alpha_{I}\right) \Theta^{I}$. Also note that Leibniz' rule holds:

$$
\delta_{j}^{n}(a b)=\sum_{i=0}^{n}\left(\begin{array}{c}
n \\
i
\end{array}\right) \delta_{j}^{i}(a) \delta_{j}^{n-i}(b)
$$

and also

$$
\Theta_{j}^{n} a=\sum_{i=0}^{n}\left(\begin{array}{c}
n \\
i
\end{array}\right) \delta_{j}^{i}(a) \Theta_{j}^{n-i}
$$

for all $i, j$, all $a, b \in R$.

The ring $R$ can also be considered as a left $T$-module with a $T$ module multiplication $*$ under which $\Theta_{j} * r=\delta_{j}(r)$ and $s * r=s r$ for all $j$, all $s, r \in R$. With this module structure, right multiplication in $R$ by a $\left(\delta_{1}, \ldots, \delta_{k}\right)$-constant is a left $T$-module endomorphism of $R$, and these are the only left $T$-module endomorphisms of $R$.

The next theorem has been proved in [5] and [11, Theorems 3.3, 3.4]. We supply a proof to indicate the difference between the $\mathbf{Q}$ algebra case and the $(\mathbf{Z} / p \mathbf{Z})$-algebra case. The case where $k=1$ was originally proved by Amitsur [1].

Theorem 2. Let $R$ be a $\mathbf{Q}$-algebra, $\delta_{1}, \ldots, \delta_{k}$ commuting derivations on $R$ and $F$ the subring of central $\left(\delta_{1}, \ldots, \delta_{k}\right)$-constants of $R$. Then the ring

$$
T=R\left[\Theta_{1}, \ldots, \Theta_{k} ; \delta_{1}, \ldots, \delta_{k}\right]
$$

is simple if and only if $R$ is $\left(\delta_{1}, \ldots, \delta_{k}\right)$-simple and no nontrivial $F$ linear combination of $\delta_{1}, \ldots, \delta_{k}$ is an inner derivation by $a\left(\delta_{1}, \ldots, \delta_{k}\right)$ constant. In this case the center of $T$ is $F$.

Proof. If $T$ is simple, $R$ is $\left(\delta_{1}, \ldots, \delta_{k}\right)$-simple and the center of $T$ is $F$ by Lemma 1. If $\alpha_{1}, \ldots, \alpha_{k} \in F$ and

$$
\left(\alpha_{1} \delta_{1}+\cdots+\alpha_{k} \delta_{k}\right)(r)=a r-r a
$$


for all $r \in R$ and some $\left(\delta_{1}, \ldots, \delta_{k}\right)$-constant $a$, then, for

$$
s=\alpha_{1} \Theta_{1}+\cdots+\alpha_{k} \Theta_{k}-a,
$$

we have $s * r=r(-a)$. So multiplication by $s$ is a left $T$-module endomorphism of $R$. Then for any $t \in T$, the commutator $s t-t s$ is in the annihilator of ${ }_{T} R$. Since $T$ is simple, ${ }_{T} R$ is faithful. This forces $s$ to be central in $T$ and so $s \in F$ by Lemma 1 . Therefore $\alpha_{i}=0$ for all $i$.

For the converse we will use induction on $k$. If $k=1$, let $A$ be a nonzero ideal of $T=R[\Theta ; \delta]$. If $n$ is the minimal order of nonzero elements of $A$, then the set

$J=\left\{a \in R \mid a \Theta^{n}+a_{n-1} \Theta^{n-1}+\cdots+a_{0} \in A\right.$ for some $\left.a_{n-1}, \ldots, a_{0} \in R\right\}$

is a nonzero $\delta$-ideal of $R$. Since $R$ is $\delta$-simple, $1 \in J$, and so we can choose $q \in A$,

$$
q=\sum_{j=0}^{n} a_{j} \Theta^{j}
$$

with $a_{n}=1$. For any $r \in R$, we have $q r-r q \in A$ of smaller order than $n$, so $q r-r q=0$. Similarly $\Theta q-q \Theta=0$, so each $a_{j}$ is a $\delta$-constant. If $n>0$,

$$
0=q r-r q=\left(n \delta(r)+a_{n-1} r-r a_{n-1}\right) \Theta^{n-1}+\text { terms of lower order, }
$$

implying $n \delta(r)+a_{n-1} r-r a_{n-1}=0$ for all $r \in R$, a contradiction. Therefore $n=0$ and $q=1 \in A$. Thus $A=T$ and $T$ is simple.

Suppose $k>1$ and the theorem is true for fewer than $k$ derivations. Now

$$
T=R\left[\Theta_{1}, \ldots, \Theta_{k} ; \delta_{1}, \ldots, \delta_{k}\right] \cong S\left[\Theta_{2}, \ldots, \Theta_{k} ; \delta_{2}, \ldots, \delta_{k}\right]
$$

where $S=R\left[\Theta_{1} ; \delta_{1}\right]$. By induction, $T$ is simple if $S$ is $\left(\delta_{2}, \ldots, \delta_{k}\right)$ simple and no nontrivial linear combination of $\delta_{2}, \ldots, \delta_{k}$ by central $\left(\delta_{2}, \ldots, \delta_{k}\right)$-constants of $S$ is an inner derivation on $S$ by a $\left(\delta_{2}, \ldots, \delta_{k}\right)$ constant of $S$. If

$$
\alpha=\sum_{i=0}^{n} a_{i} \Theta_{1}^{i}
$$

is a central $\left(\delta_{2}, \ldots, \delta_{k}\right)$-constant of $S$ with $a_{n} \neq 0$, then

$$
\delta_{j}(\alpha)=\sum_{i=0}^{n} \delta_{j}\left(a_{i}\right) \Theta_{1}^{i}=0
$$


for $j \geq 2$ and

$$
\Theta_{1} \alpha-\alpha \Theta_{1}=\sum_{i=0}^{n} \delta_{1}\left(a_{i}\right) \Theta_{1}^{i}=0 .
$$

So each $a_{j}$ is a $\left(\delta_{1}, \ldots, \delta_{k}\right)$-constant. If $n>0$, then for any $r \in R$,

$$
\begin{aligned}
0= & \alpha r-r \alpha=\left(a_{n} r-r a_{n}\right) \Theta_{1}^{n}+\left(n a_{n} \delta_{1}(r)+a_{n-1} r-r a_{n-1}\right) \Theta_{1}^{n-1} \\
& + \text { terms of lower order. }
\end{aligned}
$$

The vanishing of the $\Theta_{1}^{n}$ term yields $a_{n} \in F$, and then from the vanishing of the $\Theta_{1}^{n-1}$ term we get a contradiction. Therefore $n=0$ and all central $\left(\delta_{2}, \ldots, \delta_{k}\right)$-constants of $S$ are in $F$.

Suppose $\alpha_{2} \delta_{2}+\cdots+\alpha_{k} \delta_{k}=\delta_{b}$, the inner derivation on $S$ by the $\left(\delta_{2}, \ldots, \delta_{k}\right)$-constant $b=b_{0}+\cdots+b_{m} \Theta_{1}^{m}$, with $b_{m} \neq 0$ and the $\alpha_{i} \in F$. Since

$$
0=\left(\alpha_{2} \delta_{2}+\cdots+\alpha_{k} \delta_{k}\right)\left(\Theta_{1}\right)=b \Theta_{1}-\Theta_{1} b=\sum_{i=0}^{m}-\delta_{1}\left(b_{i}\right) \Theta_{1}^{i}
$$

and $b$ is a $\left(\delta_{2}, \ldots, \delta_{k}\right)$-constant, we have that each $b_{j}$ is a $\left(\delta_{1}, \ldots, \delta_{k}\right)$ constant. Now

$$
\delta_{b}(R)=\left(\alpha_{2} \delta_{2}+\cdots+\alpha_{k} \delta_{k}\right)(R) \subseteq R,
$$

so for all $r \in R$, the element

$$
\begin{aligned}
b r-r b= & \left(b_{m} r-r b_{m}\right) \Theta_{1}^{m}+\left(m b_{m} \delta_{1}(r)+b_{m-1} r-r b_{m-1}\right) \Theta_{1}^{m-1} \\
& + \text { terms of lower order }
\end{aligned}
$$

is in $R$. If $m \geq 2$, then $b_{m} \in F$ and we get a contradiction from the vanishing of the $\Theta_{1}^{m-1}$ term. Therefore $m \leq 1$ and $b=b_{0}+b_{1} \Theta_{1}$ (here $b_{1}$ may be 0 ). As before $b_{1} \in F$ and for any $r \in R$,

$$
\left(\alpha_{2} \delta_{2}+\cdots+\alpha_{k} \delta_{k}\right)(r)=b r-r b=b_{1} \delta_{1}(r)+b_{0} r-r b_{0} .
$$

Hence each $\alpha_{i}=0$. Therefore no nontrivial linear combination of $\delta_{2}, \ldots, \delta_{k}$ by central $\left(\delta_{2}, \ldots, \delta_{k}\right)$-constants of $S$ is an inner derivation on $S$ by a $\left(\delta_{2}, \ldots, \delta_{k}\right)$-constant of $S$.

Suppose $S$ has a nonzero $\left(\delta_{2}, \ldots, \delta_{k}\right)$-ideal $B$. Let $l$ be the minimal order of nonzero elements of $B$. Then the set $J=\left\{a \in R \mid a \Theta_{1}^{l}+a_{l-1} \Theta_{1}^{l-1}+\cdots+a_{0} \in B\right.$ for some $\left.a_{l-1}, \ldots, a_{0} \in R\right\}$ is a nonzero $\left(\delta_{1}, \ldots, \delta_{k}\right)$-ideal of $R$. Since $R$ is $\left(\delta_{1}, \ldots, \delta_{k}\right)$-simple, $1 \in J$, and so there is an operator

$$
s=\Theta_{1}^{l}+s_{l-1} \Theta_{1}^{l-1}+\cdots+s_{0} \in B
$$


Since $\delta_{j}(s) \in B$ for $j \geq 2$ and $\delta_{1}(s)=\Theta_{1} s-s \Theta_{1} \in B$, we have $\delta_{j}(s)=0$ for all $j$ since these operators have lower order than $l$. Therefore each $s_{j}$ is a $\left(\delta_{1}, \ldots, \delta_{k}\right)$-constant. Also, if $l>0$, for any $r \in R$, we obtain

$$
0=s r-r s=\left(l \delta_{1}(r)+s_{l-1} r-r s_{l-1}\right) \Theta_{1}^{l-1}+\text { terms of lower order }
$$

and the vanishing of the $\Theta_{1}^{l-1}$ term yields a contradiction. Therefore $l=0$ and $1 \in B$ proving that $S$ is $\left(\delta_{2}, \ldots, \delta_{k}\right)$-simple.

The induction hypothesis is now verified, and thus $T$ is simple.

When $R$ is a $(\mathbf{Z} / p \mathbf{Z})$-algebra for some prime $p$, if $\delta$ is a derivation on $R$, then

$$
\delta^{p}, \delta^{p^{2}}, \ldots
$$

are all derivations on $R$. This complicates the simplicity criteria for the differential operator ring in the positive characteristic case.

Lemma 3. Let $R$ be a $(\mathbf{Z} / p \mathbf{Z})$-algebra, $p$ a prime, $\delta_{1}, \ldots, \delta_{k}$ commuting derivations on $R$ and $F$ the subring of central $\left(\delta_{1}, \ldots, \delta_{k}\right)$-constants of $R$. Assume that no nontrivial $F$-linear combination of

$$
\delta_{1}, \delta_{1}^{p}, \delta_{1}^{p^{2}}, \ldots
$$

is an inner derivation by $a\left(\delta_{1}, \ldots, \delta_{k}\right)$-constant. Set $S=R\left[\Theta_{1} ; \delta_{1}\right]$ and let $b \in S$ be $a\left(\delta_{1}, \ldots, \delta_{k}\right)$-constant.

(a) If $b r-r b \in R$ for all $r \in R$, then

$$
b=a+\sum_{j=0}^{m} a_{j} \Theta_{1}^{p^{\prime}}
$$

for some $\left(\delta_{1}, \ldots, \delta_{k}\right)$-constant $a \in R$ and some $a_{0}, \ldots, a_{m} \in F$.

(b) If $b$ is central in $S$, then $b \in F$.

Proof. (a) Write out

$$
b=\sum_{i=0}^{n} b_{i} \Theta_{1}^{i},
$$

and suppose $b_{n} \neq 0$. Since $b$ is a $\left(\delta_{1}, \ldots, \delta_{k}\right)$-constant, each $b_{i}$ is a $\left(\delta_{1}, \ldots, \delta_{k}\right)$-constant. From assumption (a), if $n>0$ we get $b_{n} \in F$. If $n \leq 1$, we are done. If $n>1$ and if $p$ does not divide $n$, then, for all $r \in R$,

$$
\begin{aligned}
b r-r b= & \left(b_{n} r-r b_{n}\right) \Theta_{1}^{n}+\left(n b_{n} \delta_{1}(r)+b_{n-1} r-r b_{n-1}\right) \Theta_{1}^{n-1} \\
& + \text { terms of lower order. }
\end{aligned}
$$


Since $b r-r b \in R$, we get a contradiction from the vanishing of the $\Theta_{1}^{n-1}$ term.

If $p \mid n$, set

$$
t=\max \left\{i \mid b_{i} \neq 0 \text { and } p \text { does not divide } i\right\}
$$

or 0 if no such $i$ exists. Let

$$
A=\left\{j \mid b_{j} \neq 0 \text { and } n \geq j>t\right\} .
$$

Note that $n \in A$, and that for $j \in A$, we have

$$
j=p^{l(j)} m_{j}
$$

with $\left(m_{j}, p\right)=1$ and $l(j) \geq 1$. Let

$$
s=\max \left(\{t-1\} \cup\left\{p^{l(j)}\left(m_{j}-1\right) \mid j \in A\right\}\right)
$$

and

$$
C=\left\{j \in A \mid p^{l(j)}\left(m_{j}-1\right)=s\right\} \cup\{t \text { if } t-1=s\} .
$$

If $t \in C$, let $m_{t}=t$ and $l(t)=0$, so that each $j \in C$ can be written as

$$
j=p^{l(j)} m_{j}
$$

Let

$$
B=\left\{b_{j} \mid j \in C\right\} .
$$

Since $b r-r b \in R$ for all $r \in R$, we have $B \subseteq F$.

If $s>0$, evaluating the coefficient of $\Theta_{1}^{s}$ in $b r-r b$, we have

$$
\sum_{j \in C} m_{j} b_{j} \delta_{1}^{p^{(j)}}(r)+b_{s} r-r b_{s}=0
$$

for all $r \in R$. Since $m_{j} b_{j} \neq 0$ for all $j \in C$, this gives a contradiction. Therefore $s=0$, and hence $t \leq 1$ and $m_{j}=1$ for all $j \in A$. This proves (a).

(b) If $b$ is central, then $b r-r b=0 \in R$ for all $r \in R$, so by part (a) we have

$$
b=a+\sum_{j=0}^{m} a_{j} \Theta_{1}^{p^{\prime}}
$$

for some $\left(\delta_{1}, \ldots, \delta_{k}\right)$-constant $a \in R$ and some $a_{0}, \ldots, a_{m} \in F$. But

$$
0=b r-r b=\sum_{j=0}^{m} a_{j} \delta_{1}^{p^{\prime}}(r)+a r-r a
$$

for all $r \in R$, implying $a_{j}=0$ for all $j$. Then $a$ is central in $R$ and so $b \in F$. 
The next theorem was proved for $k=1$ in [8, Thm. 4.1.6]. The case where $k=1$ and $R$ is commutative was considered in [2, Thm. 3.2a] and [3, Thm. 2.3]. There are related results in [5] and [11, Thm. 3.5]; in particular, Voskoglou proves that the given conditions are necessary for $T$ to be simple.

THEOREM 4. Let $R$ be a $(\mathbf{Z} / p \mathbf{Z})$-algebra, $p$ a prime, $\delta_{1}, \ldots, \delta_{k}$ commuting derivations on $R$ and $F$ the subring of central $\left(\delta_{1}, \ldots, \delta_{k}\right)$ constants of $R$. Then the ring

$$
T=R\left[\Theta_{1}, \ldots, \Theta_{k} ; \delta_{1}, \ldots, \delta_{k}\right]
$$

is simple if and only if $R$ is $\left(\delta_{1}, \ldots, \delta_{k}\right)$-simple and no nontrivial $F$ linear combination of

$$
\left\{\delta_{i}^{p^{\prime}} \mid i=1, \ldots, k, j=0,1, \ldots\right\}
$$

is an inner derivation by a $\left(\delta_{1}, \ldots, \delta_{k}\right)$-constant. In this case the center of $T$ is $F$.

Proof. If $T$ is simple, $R$ is $\left(\delta_{1}, \ldots, \delta_{k}\right)$-simple and the center of $T$ is $F$ by Lemma 1. If there are some $\alpha_{i j} \in F$ such that

$$
\sum \alpha_{i j} \delta_{i}^{p^{\prime}}
$$

is an inner derivation by the $\left(\delta_{1}, \ldots, \delta_{k}\right)$-constant $a$, then

$$
s=\sum \alpha_{i j} \Theta_{i}^{p^{\prime}}-a
$$

acts on ${ }_{T} R$ as right multiplication by $-a$. For any $t \in T$, we get that $s t-t s$ is in the annihilator of ${ }_{T} R$. Then $T$ being simple implies $s$ is central in $T$. By Lemma 1, we have $s \in F$ and so each $\alpha_{i j}=0$.

For the converse we use induction on $k$. If $k=1$ and $A$ is a nonzero ideal of $T=R[\Theta ; \delta]$ with $n$ the minimal order of nonzero elements of $A$, the set

$J=\left\{a \in R \mid a \Theta^{n}+a_{n-1} \Theta^{n-1}+\cdots+a_{0} \in A\right.$ for some $\left.a_{n-1}, \ldots, a_{0} \in R\right\}$

is a nonzero $\delta$-ideal of $R$. Since $R$ is $\delta$-simple, $1 \in J$, and we can pick $q \in A$,

$$
q=\sum_{i=0}^{n} a_{i} \Theta^{i}
$$

with $a_{n}=1$. Since $\Theta q-q \Theta \in A$ and this operator is of lower order than $n$, we get $\Theta q-q \Theta=0$ and so each $a_{i}$ is a $\delta$-constant. Also 
$q r-r q=0$ for all $r \in R$, and so $q$ is central in $T$. By Lemma 3, we have $q \in F$. Therefore $n=0$ and $1 \in A$, and so $T$ is simple.

Suppose $k>1$ and the theorem is true for fewer than $k$ derivations. Now

$$
T=R\left[\Theta_{1}, \ldots, \Theta_{k} ; \delta_{1}, \ldots, \delta_{k}\right] \cong S\left[\Theta_{2}, \ldots, \Theta_{k} ; \delta_{2}, \ldots, \delta_{k}\right]
$$

where $S=R\left[\Theta_{1} ; \delta_{1}\right]$. By induction $T$ is simple if $S$ is $\left(\delta_{2}, \ldots, \delta_{k}\right)$ simple and no nontrivial linear combination of

$$
\left\{\delta_{i}^{p^{j}} \mid i=2, \ldots, k, j=0,1, \ldots\right\}
$$

by central $\left(\delta_{2}, \ldots, \delta_{k}\right)$-constants of $S$ is an inner derivation by a $\left(\delta_{2}, \ldots, \delta_{k}\right)$-constant of $S$.

Suppose there exist $\alpha_{i j}$, with $i>1$, central $\left(\delta_{2}, \ldots, \delta_{k}\right)$-constants of $S$ such that

$$
\sum \alpha_{i j} \delta_{i}^{p^{\prime}}
$$

is inner by the $\left(\delta_{2}, \ldots, \delta_{k}\right)$-constant

$$
b=\sum_{i=0}^{n} b_{i} \Theta_{1}^{i} .
$$

Since the $\alpha_{i j}$ are central in $S$, they are also $\delta_{1}$-constants and so each $\alpha_{i j} \in F$ by Lemma 3. For all $r \in R$,

$$
b r-r b=\sum \alpha_{i j} \delta_{i}^{p^{\prime}}(r) \in R
$$

So, by Lemma 3,

$$
b=a+\sum_{j=0}^{m} a_{j} \Theta_{1}^{p^{\prime}}
$$

for some $\left(\delta_{1}, \ldots, \delta_{k}\right)$-constant $a \in R$ and some $a_{0}, \ldots, a_{m} \in F$. Then, for any $r \in R$,

$$
\sum \alpha_{i j} \delta_{i}^{p^{j}}(r)-\sum_{j=0}^{m} a_{j} \delta_{1}^{p^{\jmath}}(r)=a r-r a,
$$

and so each $\alpha_{i j}=0$.

If $S$ has a nonzero $\left(\delta_{2}, \ldots, \delta_{k}\right)$-ideal $B$, let $n$ be the minimal order of nonzero elements of $B$. Then the set

$J=\left\{a \in R \mid a \Theta_{1}^{n}+a_{n-1} \Theta_{1}^{n-1}+\cdots+a_{0} \in B\right.$ for some $\left.a_{n-1}, \ldots, a_{0} \in R\right\}$

is a nonzero $\left(\delta_{1}, \ldots, \delta_{k}\right)$-ideal of $R$. Therefore there is a $q \in B$ with

$$
q=\sum_{i=0}^{n} a_{i} \Theta_{1}^{i}
$$


and $a_{n}=1$. Also the operators $\Theta_{1} q-q \Theta_{1}=\delta_{1}(q)$, as well as $\delta_{i}(q)$ for $i>1$ and $q r-r q$ for all $r \in R$ are in $B$, have lower order than $n$, and so are 0 . Therefore $q$ is a central $\left(\delta_{1}, \ldots, \delta_{k}\right)$-constant of $S$ and so $q \in F$ by Lemma 3 . Then $n=0$ and $1 \in B$, proving that $S$ is $\left(\delta_{2}, \ldots, \delta_{k}\right)$-simple.

The induction hypothesis is now established, and therefore $T$ is simple.

As an example to satisfy the hypotheses of Theorem 4 , let $K$ be a field of characteristic $p>0$ and let $\left\{x_{i j} \mid i=1, \ldots, k, j=1,2, \ldots\right\}$ be independent indeterminates over $K$. Set $R=K\left(\left\{x_{i j}\right\}\right)$. Define commuting $K$-linear derivations $\delta_{1}, \ldots, \delta_{k}$ on $R$ by

$$
\delta_{i}\left(x_{i j}\right)=x_{i, j+1} \text { and } \delta_{i}\left(x_{m j}\right)=0
$$

for all $m \neq i$.

First we show the set

$$
\left\{\delta_{i}^{j} \mid i=1, \ldots, k, j=1,2, \ldots\right\}
$$

is linearly independent over $R$. Suppose there exists some $\alpha_{i j} \in R$ such that

$$
\sum \alpha_{i j} \delta_{i}^{j}=0
$$

For fixed $n$, pick $l$ such that no term $x_{n, l+i}$, for $i>0$, is involved in any of the $\alpha_{n, j}$. Then

$$
0=\sum \alpha_{i j} \delta_{i}^{j}\left(x_{n l}\right)=\sum_{j} \alpha_{n j} x_{n, l+j}
$$

which implies each $\alpha_{n j}=0$. Since $n$ was arbitrary we have $\alpha_{i j}=0$ for all $i, j$.

Therefore the set

$$
\left\{\delta_{i}^{p^{j}} \mid i=1, \ldots, k, j=0,1, \ldots\right\}
$$

is linearly independent over the central $\left(\delta_{1}, \ldots, \delta_{k}\right)$-constants of $R$. As $R$ is simple, the differential operator ring $T$ is simple.

2. A finite Schmidt higher derivation (S.h.d.) on a ring $R$ is a set of additive maps $d_{0}, d_{1}, \ldots, d_{k}$ on $R$ such that $d_{0}$ is the identity map and

$$
d_{i}(a b)=\sum_{j=0}^{i} d_{j}(a) d_{i-j}(b)
$$


for all $a, b \in R$ and $i=1, \ldots, k$. Infinite S.h.d.'s were first used in [4] and [10]. For example, if $\delta$ is a derivation on a $Q$-algebra $R$, then the maps

$$
d_{i}=\delta^{i} / i !
$$

define a S.h.d. on $R$. For another example, let $K$ be any field and $K[x]$ a polynomial ring over $K$. Define $K$-linear maps $d_{0}, d_{1}, \ldots, d_{k}$ on $K[x]$ such that

$$
d_{i}\left(x^{j}\right)=\left(\begin{array}{l}
j \\
i
\end{array}\right) x^{j-i}
$$

for all $i, j$. Then $d_{0}, d_{1}, \ldots, d_{k}$ is a S.h.d. on $K[x]$, with $d_{0}=$ identity map and $d_{1}=d / d x$. If $\operatorname{char}(K)=2$, then

$$
d_{2}\left(x^{2 n}\right)=n x^{2 n-2}
$$

and

$$
d_{2}\left(x^{2 n+1}\right)=n x^{2 n-1} .
$$

Let $d_{0}, d_{1}, \ldots, d_{k}$ be a S.h.d. on a ring $R$ and observe that $d_{i}(1)=0$ for $i=1, \ldots, k$. A $\left(d_{0}, d_{1}, \ldots, d_{k}\right)$-ideal of $R$ is any ideal $A$ of $R$ such that $d_{i}(A) \subseteq A$ for all $i$. The ring $R$ is said to be $\left(d_{0}, d_{1}, \ldots, d_{k}\right)$ simple if $R$ is nonzero and the only $\left(d_{0}, \ldots, d_{k}\right)$-ideals of $R$ are 0 and $R$. The elements $r \in R$ such that $d_{i}(r)=0$ for all $i>0$ are called $\left(d_{1}, \ldots, d_{k}\right)$-constants and form a subring of $R$. For $d_{0}, d_{1}, \ldots, d_{k}$ a commuting S.h.d. on $R$, we can form a differential operator ring over $R$, analogous to the differential operator rings of the previous section, as in the following theorem. We first fix some notation.

Let

$$
\begin{aligned}
& M=\{(l(0), \ldots, l(k) \mid l(0)=-\infty \text { and } \\
& \qquad l(i) \text { is a nonnegative integer for } i>0\}
\end{aligned}
$$

and observe that $M$ is an additive semigroup with zero element $e(0)=$ $(-\infty, 0, \ldots, 0)$. For $i>0$, let $e(i)=(l(0), \ldots, l(k)) \in M$ with $l(i)=1$ and $l(j)=0$ for all other $j>0$. We have for $L=(l(0), \ldots, l(k)) \in M$ and a nonnegative integer $j$,

$$
L+j e(i)=(l(0), \ldots, l(i)+j, \ldots, l(k))
$$

for $i>0$, while

$$
L+j e(0)=L .
$$


THEOREM 5. Let $d_{0}, d_{1}, \ldots, d_{k}$ be a commuting S.h.d. on $R$. Then there is a differential operator ring $S$ generated by $R$ and elements $D_{0}, D_{1}, \ldots, D_{k}$ with relations

$$
D_{i} D_{j}=D_{j} D_{i}, \quad D_{0}=1, \quad D_{j} r=\sum_{i=0}^{j} d_{i}(r) D_{j-i}
$$

for all $i, j$ and all $r \in R$, such that $S$ is free as a left $R$-module with the monomials

$$
D_{k}^{n(k)} \cdots D_{1}^{n(1)}
$$

for $n(i) \geq 0$ forming a basis.

Proof. Let $U$ be a free left $R$-module with basis

$$
\left\{u_{L} \mid L=(l(0), \ldots, l(k)) \in M\right\} .
$$

Let

$$
E=\operatorname{End}_{\mathbf{Z}}(U)
$$

There is an injective ring map $R \rightarrow E$ under which any element $r \in R$ corresponds to left multiplication by $r$ on $U$. We identify $R$ with its image under this map so that $R$ is now a subring of $E$.

Since $U$ is the direct sum of the subgroups $R u_{L}$, we may define maps $D_{j} \in E$, for $0 \leq j \leq k$, such that

$$
D_{j}\left(r u_{L}\right)=\sum_{i=0}^{j} d_{j}(r) u_{L+e(j-i)}
$$

for $r \in R$. Let $S$ be the subring of $E$ generated by $D_{0}, D_{1}, \ldots, D_{k}$ and $R$.

Given $r \in R$ and $L \in M$,

$$
\begin{aligned}
D_{i} D_{j}\left(r u_{L}\right) & =\sum_{m=0}^{i} \sum_{n=0}^{j} d_{m}\left(d_{n}(r)\right) u_{L+e(j-n)+e(i-m)} \\
& =\sum_{n=0}^{j} \sum_{m=0}^{i} d_{n}\left(d_{m}(r)\right) u_{L+e(i-m)+e(j-n)}=D_{j} D_{i}\left(r u_{L}\right) .
\end{aligned}
$$

Thus $D_{i} D_{j}=D_{j} D_{i}$ for all $i, j$.

Given $r, s \in R$,

$$
\begin{aligned}
D_{j}\left(r s u_{L}\right) & =\sum_{i=0}^{j} d_{i}(r s) u_{L_{+e}(j-i)}=\sum_{i=0}^{j}\left(\sum_{n=0}^{i} d_{i-n}(r) d_{n}(s)\right) u_{L+e(j-i)} \\
& =\sum_{i=0}^{j} \sum_{n=0}^{j-i} d_{i}(r) d_{n}(s) u_{L+e(j-i-n)}=\sum_{i=0}^{j} d_{i}(r) D_{j-i}\left(s u_{L}\right) .
\end{aligned}
$$


Thus

$$
D_{j} r=\sum_{i=0}^{j} d_{i}(r) D_{j-i}
$$

It follows that if

$$
D^{L}=D_{k}^{l(k)} \cdots D_{1}^{l(1)}
$$

for $L=(l(0), \ldots, l(k)) \in M$, then

$$
\sum_{L \in M} R D^{L}
$$

is closed under multiplication and so

$$
S=\sum_{L \in M} R D^{L}
$$

Thus $S$ is generated as a left $R$-module by

$$
\left\{D^{L} \mid L \in M\right\} \text {. }
$$

Since $d_{i}(1)=0$ for $i>0$, we have

$$
D_{j} u_{L}=u_{L+e(j)} \quad \text { and } \quad D_{j}^{i} u_{L}=u_{L+i e(j)} .
$$

To show the $D^{L}$ are left linearly independent over $R$, let $r_{0}, \ldots, r_{n} \in$ $R$ such that

$$
r_{0}+r_{1} D^{L(1)}+\cdots+r_{n} D^{L(n)}=0
$$

for distinct $L(i) \in M$. Then

$0=\left(r_{0}+r_{1} D^{L(1)}+\cdots+r_{n} D^{L(n)}\right) u_{e(0)}=r_{0} u_{e(0)}+r_{1} u_{L(1)}+\cdots+r_{n} u_{L(n)}$, implying $r_{0}=r_{1}=\cdots=r_{n}=0$. Thus $S$ is free as a left $R$-module with the monomials $D^{L}$ forming a basis.

We denote the differential operator ring constructed in Theorem 5 by

$$
S=R\left[D_{1}, \ldots, D_{k} ; d_{0}, d_{1}, \ldots, d_{k}\right]
$$

It is also convenient to define leading coefficients for elements of $S$. For $L=(l(0), \ldots, l(k)) \in M$, set

$$
|L|=l(1)+2 l(2)+\cdots+k l(k) \text { and } D^{L}=D_{k}^{l(k)} \cdots D_{1}^{l(1)}
$$

and call $L$ the weighted order of $D^{L}$. Let $N=(n(0), \ldots, n(k)) \in M$. We say the weighted order of $D^{N}$ is greater than that of $D^{L}$ if $|N|>|L|$ or, if $|N|=|L|$, if there exists $j \in\{1, \ldots, k\}$ such that $n(i)=l(i)$ for $i>j$ and $n(j)>l(j)$. Otherwise the weighted order of $D^{N}$ is less than or equal to that of $D^{L}$. 
For nonzero $s \in S$, the leading coefficient of $s$ is the coefficient of the monomial of highest weighted order in $s$ with nonzero coefficient. We will usually denote an element $s \in S$ by

$$
s=\sum \alpha_{L} D^{L}
$$

with $\alpha_{L} \in R$. If $\alpha_{J}$ is the leading coefficient of $s$, the weighted order of $s$ is said to be $J$. One should note that for nonzero $t, s \in S$, the leading coefficient of $t s$ is the product of the leading coefficients unless that product is zero. Although we could have defined order as in the last section, weighted order is useful in Theorem 11.

Before developing criteria for the simplicity of these rings, we note a case when $S$ is noetherian and we give a construction of a S.h.d. from derivations and vise versa.

THEOREM 6. Let $d_{0}, d_{1}, \ldots, d_{k}$ be a commuting S.h.d. on a ring $R$. If $R$ is right (left) noetherian, the ring

$$
S=R\left[D_{1}, \ldots, D_{k} ; d_{0}, d_{1}, \ldots, d_{k}\right]
$$

is right (left) noetherian.

Proof. Set

$$
T=R\left[D_{1}, \ldots, D_{k-1} ; d_{0}, d_{1}, \ldots, d_{k-1}\right]
$$

Note that for $t \in T$, we have $\delta(t)=D_{k} t-t D_{k} \in T$. Therefore, $\delta$ defines a derivation on $T$. Also $S \cong T[\Theta ; S]$. Then, by induction on $k$, and by [9, Thm. 2, p. 65], we have $R$ right (left) noetherian implies $S$ is right(left) noetherian.

For the rest of this section, we fix some notation. Given derivations $\delta_{1}, \ldots, \delta_{r}$ and $i=1, \ldots, r$, let

$$
\delta_{(i, r)}=\sum \delta_{j(1)} \cdots \delta_{j(i)}
$$

where the sum is over all formally distinct $i$-tuples $(j(1), \ldots, j(i))$ with each $j(l)$ a positive integer and $j(1)+\cdots+j(i)=r$. For example,

$$
\delta_{(2,4)}=\delta_{1} \delta_{3}+\delta_{3} \delta_{1}+\delta_{2} \delta_{2}
$$

We define $\Theta_{(i, r)}$ similarly. Also, given a S.h.d. $d_{0}, d_{1}, \ldots, d_{k}$, we define $d_{(i, r)}$ and $D_{(i, r)}$ in a similar manner. 
LEMMA 7. Let $k \in \mathbf{N}$, let $R$ be a ring in which $k$ ! is invertible, let $\delta_{1}, \ldots, \delta_{k}$ be derivations on $R$, let $R[t]$ be a polynomial ring over $R$, and set

$$
R[s]=R[t] /\left(t^{k+1}\right) .
$$

Extend each $\delta_{i}$ to a derivation on $R[s]$ where $\delta_{i}(s)=0$, and set

$$
\partial=s \delta_{1}+s^{2} \delta_{2}+\cdots+s^{k} \delta_{k} .
$$

(a) $\partial^{n}=\sum_{j=0}^{k-n} s^{n+j} \delta_{(n, n+j)}$ for $1 \leq n \leq k$.

(b) $\sigma=\sum_{n=0}^{k} \partial^{n} / n$ ! is a ring endomorphism of $R[s]$.

Proof. (a) If $n=1$,

$$
\sum_{j=0}^{k-1} s^{j+1} \delta_{(1, j+1)}=\sum_{j=0}^{k-1} s^{j+1} \delta_{j+1}=\partial .
$$

Suppose $n>1$ and (a) is true for $n-1$. Then

$$
\begin{aligned}
\partial^{n} & =\partial^{n-1} \partial=\left(\sum_{j=0}^{k-n+1} s^{n-1+j} \delta_{(n-1, n-1+j)}\right)\left(s \delta_{1}+\cdots+s^{k} \delta_{k}\right) \\
& =\sum_{i=0}^{k-n} s^{n+i}\left(\sum_{j=0}^{i} \delta_{(n-1, n+j-1)} \delta_{i-j+1}\right)=\sum_{i=0}^{k-n} s^{n+i} \delta_{(n, n+i)} .
\end{aligned}
$$

(b) Set $d=\delta_{1}+s \delta_{2}+\cdots+s^{k-1} \delta_{k}$. Then $\partial=s d$ and $d$ is a derivation on $R[s]$. For $a, b \in R[s]$,

$$
\begin{aligned}
\sigma(a) \sigma(b) & =\left(\sum_{n=0}^{k} s^{n} d^{n}(a) / n !\right)\left(\sum_{i=0}^{k} s^{i} d^{i}(b) / i !\right) \\
& =\sum_{n=0}^{k} s^{n}\left(\sum_{i=0}^{n} d^{n-i}(a) d^{i}(b) /(n-i) ! i !\right) \\
& =\sum_{n=0}^{k} s^{n}\left(\sum_{i=0}^{n}\left(\begin{array}{c}
n \\
i
\end{array}\right) d^{n-i}(a) d^{i}(b) / n !\right) \\
& =\sum_{n=0}^{k} s^{n} d^{n}(a b) / n !=\sigma(a b) .
\end{aligned}
$$

Clearly $\sigma$ is additive, so $\sigma$ is a ring endomorphism. 
LEMMA 8. Let $k \in \mathbf{N}$, let $R$ be a ring in which $k$ ! is invertible, let $d_{0}, d_{1}, \ldots, d_{k}$ be a S.h.d. on $R$, let $R[t]$ be a polynomial ring over $R$, and set

$$
R[s]=R[t] /\left(t^{k+1}\right)
$$

Extend $d_{0}, d_{1}, \ldots, d_{k}$ to a S.h.d. on $R[s]$ where $d_{i}(s)=0$ for $i=1, \ldots, k$.

(a) $\phi=\sum_{i=0}^{k} s^{i} d_{i}$ is a ring endomorphism of $R[s]$.

(b) If $\rho=\phi-1$, then

$$
\rho^{n}(a b)=\sum_{i=0}^{n} \sum_{j=0}^{n-i}\left(\begin{array}{c}
n \\
i
\end{array}\right)\left(\begin{array}{c}
n-i \\
j
\end{array}\right) \rho^{n-i}(a) \rho^{n-j}(b)
$$

for $1 \leq n \leq k$ and all $a, b \in R[s]$.

(c) $\rho^{n}=\sum_{j=0}^{k-n} s^{n+j} d_{(n, n+j)}$ for $1 \leq n \leq k$.

Proof. (a) Clearly $\phi$ is additive. For $a, b \in R$,

$$
\begin{aligned}
\phi(a) \phi(b) & =\left(\sum_{i=0}^{k} s^{i} d_{i}(a)\right)\left(\sum_{j=0}^{k} s^{j} d_{j}(b)\right) \\
& =\sum_{i=0}^{k} s^{i}\left(\sum_{j=0}^{i} d_{j}(a) d_{i-j}(b)\right) \\
& =\sum_{i=0}^{k} s^{i} d_{i}(a b)=\phi(a b) .
\end{aligned}
$$

Hence $\phi$ is a ring endomorphism.

(b) If $n=1$,

$$
\begin{aligned}
& \sum_{i=0}^{1} \sum_{j=0}^{1-i}\left(\begin{array}{l}
1 \\
i
\end{array}\right)\left(\begin{array}{c}
1-i \\
j
\end{array}\right) \rho^{1-i}(a) \rho^{1-j}(b) \\
& \quad=\rho(a) \rho(b)+\rho(a) b+a \rho(b) \\
& \quad=(\phi(a)-a)(\phi(b)-b)+(\phi(a)-a) b+a(\phi(b)-b) \\
& \quad=\phi(a) \phi(b)-a b=\phi(a b)-a b=\rho(a b) .
\end{aligned}
$$


Suppose $n>1$ and (b) is true for $i<n$. Then

$$
\begin{aligned}
& \rho^{n}(a b)= \rho \rho^{n-1}(a b) \\
&= \rho\left(\sum_{i=0}^{n-1} \sum_{j=0}^{n-1-i}\left(\begin{array}{c}
n-1 \\
i
\end{array}\right)\left(\begin{array}{c}
n-1-i \\
j
\end{array}\right) \rho^{n-1-i}(a) \rho^{n-1-j}(b)\right) \\
&= \sum_{i=0}^{n-1} \sum_{j=0}^{n-1-i}\left(\begin{array}{c}
n-1 \\
i
\end{array}\right)\left(\begin{array}{c}
n-1-i \\
j
\end{array}\right) \\
& \times\left[\rho^{n-i}(a) \rho^{n-j}(b)+\rho^{n-i}(a) \rho^{n-j-1}(b)+\rho^{n-i-1}(a) \rho^{n-j}(b)\right] \\
&= \sum_{j=0}^{n}\left[\left(\begin{array}{c}
n-1 \\
j
\end{array}\right)+\left(\begin{array}{c}
n-1 \\
j-1
\end{array}\right)\right] \rho^{n}(a) \rho^{n-j}(b) \\
&+\sum_{i=1}^{n}\left[\left(\begin{array}{c}
n-1 \\
i
\end{array}\right)+\left(\begin{array}{c}
n-1 \\
i-1
\end{array}\right)\right] \rho^{n-i}(a) \rho^{n}(b) \\
&+\sum_{i=1}^{n} \sum_{j=1}^{n-i}\left[\left(\begin{array}{c}
n-1 \\
i
\end{array}\right)\left(\begin{array}{c}
n-1-i \\
j
\end{array}\right)+\left(\begin{array}{c}
n-1 \\
i
\end{array}\right)\left(\begin{array}{c}
n-1-i \\
j-1
\end{array}\right)\right. \\
&=\left.\sum_{i=0}^{n} \sum_{j=0}^{n-i}\left(\begin{array}{c}
n \\
i
\end{array}\right)\left(\begin{array}{c}
n-1 \\
i-1
\end{array}\right)\left(\begin{array}{c}
n-i \\
j
\end{array}\right)\right] \rho^{n-i}(a) \rho^{n-j}(b) \\
& j
\end{aligned}
$$

(c) Since $\rho=\phi-1=s d_{1}+\cdots+s^{k} d_{k}$, the proof is similar to Lemma $7(\mathrm{a})$.

LEMMA 9. For integers $l, m>0$,

$$
\sum_{i=l}^{l+m}(-1)^{i+1}\left(\begin{array}{c}
i \\
i-l
\end{array}\right)\left(\begin{array}{c}
l \\
i-m
\end{array}\right) / i=0 .
$$

Proof.

$$
\begin{aligned}
& \sum_{i=l}^{l+m}(-1)^{i+1}\left(\begin{array}{c}
i \\
i-l
\end{array}\right)\left(\begin{array}{c}
l \\
i-m
\end{array}\right) / i \\
& \quad=\sum_{j=0}^{m}(-1)^{j+l+1}\left(\begin{array}{c}
j+l \\
j
\end{array}\right)\left(\begin{array}{c}
l \\
j+l-m
\end{array}\right) /(j+l) \\
& \quad=\sum_{j=0}^{m}(-1)^{j+l+1}(j+l-1) ! / j !(m-j) !(j+l-m) !
\end{aligned}
$$


which is the coefficient for $x^{m}$ in the power series expansion of $f g$ where

$$
f(x)=(-1)^{l+1}(1+x)^{-l}
$$

and

$$
g(x)=(1+x)^{l} / l \text {. }
$$

Since $f g$ is constant, this coefficient is 0 for $m>0$.

THEOREM 10. Let $k \in \mathbf{N}$ and $R$ a ring in which $k$ ! is invertible.

(a) If $\delta_{1}, \ldots, \delta_{k}$ are (commuting) derivations on $R$, then the rules

$$
\begin{aligned}
& d_{0}=\text { identity map } \\
& d_{r}=\sum_{i=1}^{r} \delta_{(i, r)} / i !
\end{aligned}
$$

define a (commuting) S.h.d. $d_{0}, d_{1}, \ldots, d_{k}$ on $R$.

(b) If $d_{0}, d_{1}, \ldots, d_{k}$ is a (commuting) S.h.d. on $R$ then the rules

$$
\partial_{r}=\sum_{j=1}^{r}(-1)^{j+1} d_{(j, r)} / j
$$

define (commuting) derivations $\partial_{1}, \ldots, \partial_{k}$ on $R$.

(c) If $\delta_{1}, \ldots, \delta_{k}$ are derivations on $R$ and $d_{0}, d_{1}, \ldots, d_{k}$ the S.h.d. constructed in $(\mathrm{a})$, then the derivations $\partial_{1}, \ldots, \partial_{k}$ constructed in (b) equal $\delta_{1}, \ldots, \delta_{k}$.

(d) If $d_{0}^{\prime}, d_{1}^{\prime}, \ldots, d_{k}^{\prime}$ is a S.h.d. on $R$ and $\partial_{1}, \ldots, \partial_{k}$ the derivations constructed in (b), then the S.h.d. $d_{0}, d_{1}, \ldots, d_{k}$ constructed in (a) is equal to $d_{0}^{\prime}, d_{1}^{\prime}, \ldots, d_{k}^{\prime}$.

Proof. (a) For the ring endomorphism $\sigma$ defined in Lemma 7,

$$
\begin{aligned}
\sigma & =\sum_{n=0}^{k} \partial^{n} / n !=1+\sum_{n=1}^{k} \sum_{j=0}^{k-n} s^{n+j} \delta_{(n, n+j)} / n ! \\
& =1+\sum_{n=1}^{k} s^{n} \sum_{i=1}^{n} \delta_{(i, n)} / i !
\end{aligned}
$$

For any ring homomorphism

$$
d=\sum_{i=0}^{k} s^{i} d_{i}: R \rightarrow R[s]
$$

where $d_{i}: R \rightarrow R$, the maps $d_{0}, d_{1}, \ldots, d_{k}$ form a S.h.d. on $R$. Hence (a) is proved. 
(b) Using the map $\rho$ defined in Lemma 8, set

$$
\delta=\sum_{n=1}^{k}\left((-1)^{n+1} / n\right) \rho^{n} .
$$

We have, for any $a, b \in R[s]$,

$$
\begin{aligned}
\delta(a b) & =\sum_{n=1}^{k}\left((-1)^{n+1} / n\right) \rho^{n}(a b) \\
& =\sum_{n=1}^{k} \sum_{i=0}^{n} \sum_{j=0}^{n-1}\left((-1)^{n+1} / n\right)\left(\begin{array}{c}
n \\
i
\end{array}\right)\left(\begin{array}{c}
n-i \\
j
\end{array}\right) \rho^{n-i}(a) \rho^{n-j}(b) .
\end{aligned}
$$

Note that $\rho^{l}(a) \rho^{m}(b)=0$ whenever $l+m>k$. The coefficient of a particular term $\rho^{l}(a) \rho^{m}(b)$ above for $l \geq m>0$ and $l+m \leq k$ is

$$
\sum_{i=l}^{l+m}\left((-1)^{i+1} / i\right)\left(\begin{array}{c}
i \\
i-l
\end{array}\right)\left(\begin{array}{c}
l \\
i-m
\end{array}\right)
$$

which is 0 by Lemma 9. Similarly, for $m \geq l>0$ and $l+m \leq k$, the coefficient of $\rho^{l}(a) \rho^{m}(b)$ in the above is 0 . The coefficient of a term $\rho^{l}(a) b\left(\right.$ or $\left.a \rho^{l}(b)\right)$ is $(-1)^{l+1} / l$. Hence, $\delta(a b)=\delta(a) b+a \delta(b)$ and $\delta$ is a derivation on $R[s]$.

If $\beta$ is any derivation from $R$ to $R[s]$ and

$$
\beta=\sum_{i=0}^{k} s^{i} \beta_{i}
$$

with each $\beta_{i}: R \rightarrow R$, then each $\beta_{i}$ is a derivation on $R$. Now, by Lemma 8,

$$
\begin{aligned}
\delta & =\sum_{n=1}^{k}\left((-1)^{n+1} / n\right) \rho^{n}=\sum_{n=1}^{k} \sum_{j=0}^{k-n}\left((-1)^{n+1} / n\right) s^{n+j} d_{(n, n+j)} \\
& =\sum_{n=1}^{k} s^{n} \sum_{j=1}^{n}\left((-1)^{j+1} / j\right) d_{(j, n)}=\sum_{n=1}^{k} s^{n} \partial_{n},
\end{aligned}
$$

so each $\partial_{n}$ is a derivation on $R$.

(c) If $\partial=s \delta_{1}+\cdots+s^{k} \delta_{k}$ and $\rho=s d_{1}+\cdots+s^{k} d_{k}$, we have

$$
\sum_{n=0}^{k} \partial^{n} / n !=\rho+1
$$


as in the proof of (a) and

$$
\sum_{n=1}^{k}\left((-1)^{n+1} / n\right) \rho^{n}=\sum_{n=1}^{k} s^{n} \partial_{n}
$$

as in the proof of (b). Hence,

$$
\sum_{n=1}^{k}\left((-1)^{n+1} / n\right)\left(\sum_{i=1}^{k} \partial^{i} / i !\right)^{n}=\sum_{n=1}^{k} s^{n} \partial_{n}
$$

But

$$
\partial=\sum_{n=1}^{k}\left((-1)^{n+1} / n\right)\left(\sum_{i=1}^{k} \partial^{i} / i !\right)^{n}
$$

since the coefficient of a term $\partial^{j}$ for $j \leq k$ is the coefficient of $x^{j}$ in the power series expansion of $\log e^{x}$ and $\partial^{j}=0$ for $j>k$.

So $\partial=s \delta_{1}+\cdots+s^{k} \delta_{k}=s \partial_{1}+\cdots+s^{k} \partial_{k}$ and $\partial_{i}=\delta_{i}$ for all $i$.

(d) For $\sigma=d_{0}^{\prime}+s d_{1}^{\prime}+\cdots+s^{k} d_{k}^{\prime}$,

$$
\sigma=\sum_{n=0}^{k}\left(\sum_{i=1}^{k}\left((-1)^{i+1} / i\right)(\sigma-1)^{i}\right)^{n} / n !
$$

since the coefficient of $\sigma^{j}$ for $j \leq k$ is the coefficient of $x^{j}$ in the power series expansion of $e^{\log x}$ and $\sigma^{j}=0$ for $j>k$.

On the other hand,

$$
\sum_{i=1}^{k}\left((-1)^{i+1} / i\right)(\sigma-1)^{i}=\sum_{n=1}^{k} s^{n} \partial_{n}
$$

as in the proof of (b). If we denote this map by $\partial$, then as in the proof of (a),

$$
\sum_{n=0}^{k} \partial^{n} / n !=1+\sum_{n=1}^{k} s^{n} \sum_{i=1}^{n} \partial_{(i, n)} / i !=1+s d_{1}+\cdots+s^{k} d_{k} .
$$

Since the left hand side equals $\sigma$ (as shown above) we obtain $d_{i}^{\prime}=d_{i}$ for all $i$.

THEOREM 11. Let $k \in \mathbf{N}$, and let $R$ be a ring in which $k$ ! is invertible. Let $d_{0}, d_{1}, \ldots, d_{k}$ be a commuting S.h.d. on $R$ and $\delta_{1}, \ldots, \delta_{k}$ the corresponding commuting derivations as in Theorem 10(b). Then the rings

$S=R\left[D_{1}, \ldots, D_{k} ; d_{0}, d_{1}, \ldots, d_{k}\right]$ and $T=R\left[\Theta_{1}, \ldots, \Theta_{k} ; \delta_{1}, \ldots, \delta_{k}\right]$ 
are isomorphic. Moreover, there is an isomorphism that is the identity on $R$ and preserves leading coefficients.

Proof. Define $\bar{\Theta}_{1}, \ldots, \bar{\Theta}_{k}$ by

$$
\bar{\Theta}_{n}=\sum_{i=1}^{n}\left((-1)^{i+1} / i\right) D_{(i, n)} .
$$

Note that $\bar{\Theta}_{i} \bar{\Theta}_{j}=\bar{\Theta}_{j} \bar{\Theta}_{i}$ for all $i, j$. Let $R[t]$ be a polynomial ring over $R$ and set

$$
R[s]=R[t] /\left(t^{k+1}\right)
$$

Set

$$
D=s D_{1}+\cdots+s^{k} D_{k} \quad \text { and } \quad \rho=s d_{1}+\cdots+s^{k} d_{k}
$$

As in the proof of Lemma 7(a)

$$
D^{n}=\sum_{j=0}^{k-n} s^{n+j} D_{(n, n+j)}
$$

for $1 \leq n \leq k$. Hence

$$
\begin{aligned}
\sum_{n=1}^{k}\left((-1)^{n+1} / n\right) D^{n} & =\sum_{n=1}^{k} \sum_{j=0}^{k-n} s^{n+j}\left((-1)^{n+1} / n\right) D_{(n, n+j)} \\
& =\sum_{n=1}^{k} s^{n} \sum_{j=1}^{n}\left((-1)^{j+1} / j\right) D_{(j, n)}=\sum_{n=1}^{k} s^{n} \bar{\Theta}_{n}
\end{aligned}
$$

Now for $1 \leq n \leq k$ and $a \in R[s]$ we show that

$$
D^{n} a=\sum_{i=0}^{n} \sum_{j=0}^{n-i}\left(\begin{array}{c}
n \\
i
\end{array}\right)\left(\begin{array}{c}
n-i \\
j
\end{array}\right) \rho^{n-i}(a) D^{n-j}
$$

To see this note that

$$
D a=\rho(a) D+a D+\rho(a),
$$


which is the above with $n=1$. Then by induction,

$$
\begin{aligned}
& D^{n} a=D^{n-1} D a=D^{n-1}(\rho(a) D+a D+\rho(a)) \\
& =\sum_{i=0}^{n-1} \sum_{j=0}^{n-1-i}\left(\begin{array}{c}
n-1 \\
i
\end{array}\right)\left(\begin{array}{c}
n-1-i \\
j
\end{array}\right) \\
& \times\left(\rho^{n-i}(a) D+\rho^{n-1-i}(a) D+\rho^{n-i}(a)\right) D^{n-1-j} \\
& =\sum_{i=1}^{n} \sum_{j=1}^{n-i}\left[\left(\begin{array}{c}
n-1 \\
i
\end{array}\right)\left(\begin{array}{c}
n-1-i \\
j
\end{array}\right)+\left(\begin{array}{c}
n-1 \\
i
\end{array}\right)\left(\begin{array}{c}
n-1-i \\
j-1
\end{array}\right)\right. \\
& \left.+\left(\begin{array}{c}
n-1 \\
i-1
\end{array}\right)\left(\begin{array}{c}
n-i \\
j
\end{array}\right)\right] \rho^{n-i}(a) D^{n-j} \\
& +\sum_{j=0}^{n}\left[\left(\begin{array}{c}
n-1 \\
j
\end{array}\right)+\left(\begin{array}{c}
n-1 \\
j-1
\end{array}\right)\right] \rho^{n}(a) D^{n-j} \\
& +\sum_{i=1}^{n}\left[\left(\begin{array}{c}
n-1 \\
i
\end{array}\right)+\left(\begin{array}{c}
n-1 \\
i-1
\end{array}\right)\right] \rho^{n-i}(a) D^{n} \\
& =\sum_{i=0}^{n} \sum_{j=0}^{n-i}\left(\begin{array}{c}
n \\
i
\end{array}\right)\left(\begin{array}{c}
n-i \\
j
\end{array}\right) \rho^{n-i}(a) D^{n-j} \text {. }
\end{aligned}
$$

Set

$$
\Theta=\sum_{n=1}^{k} s^{n} \bar{\Theta}_{n}=\sum_{n=1}^{k}\left((-1)^{n+1} / n\right) D^{n} .
$$

Then for $a \in R$ we get

$$
\begin{aligned}
\Theta a & =\sum_{n=1}^{k} \sum_{i=0}^{n} \sum_{j=0}^{n-i}\left((-1)^{n+1} / n\right)\left(\begin{array}{c}
n \\
i
\end{array}\right)\left(\begin{array}{c}
n-i \\
j
\end{array}\right) \rho^{n-i}(a) D^{n-j} \\
& =a \Theta+\sum_{n=1}^{k} s^{n} \delta_{n}(a)
\end{aligned}
$$

as in the proof of Theorem 10(b). Hence,

$$
\bar{\Theta}_{n} a=a \bar{\Theta}_{n}+\delta_{n}(a),
$$

for $1 \leq n \leq k$ and all $a \in R$.

Consequently, there exists a unique ring homomorphism $\phi: T \rightarrow S$ such that $\phi$ is the identity on $R$ and $\phi\left(\Theta_{n}\right)=\bar{\Theta}_{n}$ for all $n$.

If

$$
0 \neq \sum r_{L} \Theta^{L} \in T
$$


with leading coefficient $r_{J}$, then

$$
\phi\left(\sum r_{L} \Theta^{L}\right) \in S
$$

also has leading coefficient $r_{J}$. To see this, note that the highest weighted order monomial of $\bar{\Theta}_{n}$ is $D_{n}$. Therefore

$$
\sum r_{L} \Theta^{L} \neq 0
$$

implies

$$
\phi\left(\sum r_{L} \Theta^{L}\right) \neq 0
$$

and hence $\phi$ is injective.

As in the proof of Theorem 10(d), we have

$$
\sum_{n=0}^{k}\left(\sum_{i=1}^{k}\left((-1)^{i+1} / i\right) D^{i}\right)^{n} / n !=D+1 .
$$

Therefore

$$
\begin{aligned}
D+1 & =\sum_{n=0}^{k}\left(\sum_{i=1}^{k} s^{i} \overline{\boldsymbol{\Theta}}_{i}\right)^{n} / n ! \\
& =1+\sum_{n=1}^{k}\left(\sum_{j=0}^{k-n} s^{n+j} \bar{\Theta}_{(n, n+j)}\right) / n !=1+\sum_{n=1}^{k} s^{n} \sum_{i=1}^{n} \overline{\boldsymbol{\Theta}}_{(i, n)} / i !
\end{aligned}
$$

and it follows that

$$
D_{n}=\sum_{i=1}^{n} \overline{\boldsymbol{\Theta}}_{(i, n)} / i !=\phi\left(\sum_{i=1}^{n} \Theta_{(i, n)} / i !\right)
$$

for $1 \leq n \leq k$. Hence $\phi$ is also surjective and therefore an isomorphism.

We end this section with two lemmas that allow us to extend a S.h.d.

LeMmA 12. Let $d_{0}, d_{1}, \ldots, d_{k}$ be a S.h.d. on a ring $R$ and let $C$ be a right denominator set in $R$. Then $d_{0}, d_{1}, \ldots, d_{k}$ can be uniquely extended to a S.h.d. on $R\left[C^{-1}\right]$.

Proof. Let $R[t]$ be a polynomial ring over $R$ and set

$$
R[s]=R[t] /\left(t^{k+1}\right) .
$$


Note that $d=d_{0}+s d_{1}+\cdots+s^{k} d_{k}$ defines a ring homomorphism $d: R \rightarrow R[s]$. Let $\phi: R \rightarrow R\left[C^{-1}\right]$ and $\phi^{\prime}: R[s] \rightarrow R\left[C^{-1}\right][s]$ be the natural maps. For any $c \in C$,

$$
\begin{aligned}
\phi^{\prime} d(c) & =\phi^{\prime}\left(d_{0}(c)+s d_{1}(c)+\cdots+s^{k} d_{k}(c)\right) \\
& =\phi^{\prime}(c)+s \phi^{\prime}\left(d_{1}(c)\right)+\cdots+s^{k} \phi^{\prime}\left(d_{k}(c)\right) .
\end{aligned}
$$

Since $\phi^{\prime}(c)^{-1}$ exists in $R\left[C^{-1}\right][s]$, we can inductively solve for coefficients of a right or left inverse for $\phi^{\prime}(d(c))$. Therefore, there exists $\phi^{\prime}(d(c))^{-1}$ in $R\left[C^{-1}\right][s]$ for all $c \in C$.

Hence, there exists a unique ring homomorphism

$$
d^{\prime}: R\left[C^{-1}\right] \rightarrow R\left[C^{-1}\right][s]
$$

such that $d^{\prime} \phi=\phi^{\prime} d$. If we write $d^{\prime}=d_{0}^{\prime}+s d_{1}^{\prime}+\cdots+s^{k} d_{1}^{\prime}$ with each $d_{i}^{\prime}: R\left[C^{-1}\right] \rightarrow R\left[C^{-1}\right], d_{0}^{\prime}, d_{1}^{\prime}, \ldots, d_{k}^{\prime}$ is a S.h.d. on $R\left[C^{-1}\right]$ which uniquely extends $d_{0}, d_{1}, \ldots, d_{k}$.

LemMA 13. Let $d_{0}, d_{1}, \ldots, d_{k}$ be a S.h.d. on a ring $R$. Given $f_{1}, \ldots, f_{k}$ central elements of the polynomial ring $R[x]$, there is a unique extension of $d_{0}, d_{1}, \ldots, d_{k}$ to a S.h.d. on $R[x]$ such that $d_{i}(x)=f_{i}$ for $1 \leq i \leq k$.

Proof. Let $R[t]$ be a polynomial ring over $R$ and set

$$
R[s]=R[t] /\left(t^{k+1}\right) .
$$

Then we have a ring homomorphism

$$
d=d_{0}+s d_{1}+\cdots+s^{k} d_{k}: R \rightarrow R[s] .
$$

This extends uniquely to a ring homomorphism

$$
d^{\prime}: R[x] \rightarrow R[x][s]
$$

such that

$$
d^{\prime}(x)=x+s f_{1}+\cdots+s^{k} f_{k},
$$

because $x+s f_{1}+\cdots+s^{k} f_{k}$ is central in $R[x][s]$. If

$$
d^{\prime}=d_{0}^{\prime}+s d_{1}^{\prime}+\cdots+s^{k} d_{k}^{\prime}
$$

with each $d_{i}^{\prime}: R[x] \rightarrow R[x]$, then $d_{0}^{\prime}, d_{1}^{\prime}, \ldots, d_{k}$ is a S.h.d. on $R[x]$ extending $d_{0}, d_{1}, \ldots, d_{k}$. The uniqueness is clear.

3. We develop some necessary and sufficient conditions for simplicity of differential operator rings coming from a S.h.d. The proof of the next lemma is similar to that of Lemma 1. 
LEMMA 14. Let $d_{0}, d_{1}, \ldots, d_{k}$ be a commuting S.h.d. on a ring $R$ and let $F$ be the subring of central $\left(d_{1}, \ldots, d_{k}\right)$-constants of $R$. If the ring

$$
S=R\left[D_{1}, \ldots, D_{k} ; d_{0}, d_{1}, \ldots, d_{k}\right]
$$

is simple then the center of $S$ is $F$ and $R$ is $\left(d_{0}, \ldots, d_{k}\right)$-simple.

If $d_{0}, d_{1}, \ldots, d_{k}$ is a commuting S.h.d. on a ring $R$, then $R$ can be made into a left module over the ring

$$
S=R\left[D_{1}, \ldots, D_{k} ; d_{0}, d_{1}, \ldots, d_{k}\right]
$$

using an $S$-module multiplication $*$ by which $D_{i} * r=d_{i}(r)$ and $s * r=s r$ for all $i$ and all $s, r \in R$. With this left module structure, right multiplication in $R$ by a $\left(d_{1}, \ldots, d_{k}\right)$-constant is a left $S$-module endomorphism of $R$.

THEOREM 15. Let $R$ be a Q-algebra, $d_{0}, d_{1}, \ldots, d_{k}$ a commuting S.h.d. on $R$ and $F$ the subring of central $\left(d_{1}, \ldots, d_{k}\right)$-constants of $R$. Then the ring

$$
S=R\left[D_{1}, \ldots, D_{k} ; d_{0}, d_{1}, \ldots, d_{k}\right]
$$

is simple if and only if $R$ is $\left(d_{0}, d_{1}, \ldots, d_{k}\right)$-simple and no nontrivial $F$-linear combination of

$$
\left\{d_{k}^{i(k)} \cdots d_{1}^{i(1)} \mid i(j) \geq 0 \text { and } 0<i(1)+2 i(2)+\cdots+k i(k) \leq k\right\}
$$

is an inner derivation by $a\left(d_{1}, \ldots, d_{k}\right)$-constant. In this case the center $S$ is $F$.

Proof. If $S$ is simple then the center of $S$ is $F$ and $R$ is $\left(d_{0}, \ldots, d_{k}\right)$ simple by Lemma 14. In a manner similar to that of Theorem 2, no nontrivial $F$-linear combination of

$$
\left\{d_{k}^{i(k)} \cdots d_{1}^{i(1)} \mid i(j) \geq 0 \text { and } 0<i(1)+2 i(2)+\cdots+k i(k) \leq k\right\}
$$

is an inner derivation by a $\left(d_{1}, \ldots, d_{k}\right)$-constant.

Conversely, using the construction of Theorem 10 and Theorem 11, we have

$$
S \cong T=R\left[\Theta_{1}, \ldots, \Theta_{k} ; \delta_{1}, \ldots, \delta_{k}\right]
$$

for commuting derivations $\delta_{1}, \ldots, \delta_{k}$ related to $d_{0}, d_{1}, \ldots, d_{k}$ as in Theorem 10. Then $R$ being $\left(d_{0}, d_{1}, \ldots, d_{k}\right)$-simple implies $R$ is $\left(\delta_{1}, \ldots, \delta_{k}\right)$ simple. No nontrivial $F$-linear combinations of

$$
\left\{d_{k}^{i(k)} \ldots d_{1}^{i(1)} \mid i(j) \geq 0 \text { and } 0<i(1)+2 i(2)+\cdots+k i(k) \leq k\right\}
$$


being inner by a $d_{1}, \ldots, d_{k}$-constant implies no nontrivial $F$-linear combination of $\delta_{1}, \ldots, \delta_{k}$ is inner by a $\left(\delta_{1}, \ldots, \delta_{k}\right)$-constant. Since $F$ is also the subring of central $\left(\delta_{1}, \ldots, \delta_{k}\right)$-constants of $R$, by Theorem 2 the ring $S$ is simple.

LEMMA 16. Let $d_{0}, d_{1}, \ldots, d_{k}$ be a commuting S.h.d. on a ring $R$ and I a nonzero ideal of the ring

$$
S=R\left[D_{1}, \ldots, D_{k} ; d_{0}, d_{1}, \ldots, d_{k}\right] .
$$

If $q=\sum r_{L} D^{L} \in I$, then $d_{i}(q)=\sum d_{i}\left(r_{L}\right) D^{L} \in I$ for all $i$.

Proof. If $i=1$ then $\sum d_{1}\left(r_{L}\right) D^{L}=D_{1} q-q D_{1} \in I$. If $i>1$, suppose the lemma is true for all $j<i$. Then

$$
\sum d_{i}\left(r_{L}\right) D^{L}=D_{i} q-\sum_{j=0}^{i-1} d_{j}(q) D_{i-j} \in I \text {. }
$$

THEOREM 17. Let $d_{0}, d_{1}, \ldots, d_{k}$ be a commuting S.h.d. on a ring $R$ and $F$ the subring of central $\left(d_{1}, \ldots, d_{k}\right)$-constants of $R$. Then the ring

$$
S=R\left[D_{1}, \ldots, D_{k} ; d_{0}, d_{1}, \ldots, d_{k}\right]
$$

is simple if and only if $R$ is $\left(d_{0}, d_{1}, \ldots, d_{k}\right)$-simple and no nontrivial $F$-linear combination of

$$
\left\{d_{k}^{n(k)} \cdots d_{1}^{n(1)} \mid n(i) \geq 0 \text { and } n(k)+\cdots+n(1)>0\right\}
$$

is an inner derivation by a $\left(d_{1}, \ldots, d_{k}\right)$-constant. In this case the center of $S$ is $F$.

Proof. If $S$ is simple, the proof is similar to that of Theorem 15. Conversely, assume that $R$ is $\left(d_{0}, d_{1}, \ldots, d_{k}\right)$-simple and no nontrivial $F$-linear combination of

$$
\left\{d_{k}^{n(k)} \cdots d_{1}^{n(1)} \mid n(i) \geq 0 \text { and } n(k)+\cdots+n(1)>0\right\}
$$

is inner by a $\left(d_{1}, \ldots, d_{k}\right)$-constant.

If $A$ is a nonzero ideal of $S$, let $J$ be the minimal weighted order of nonzero elements of $A$. Using Lemma 16, the set of 0 together with those $r \in R$ that are leading coefficient of elements of $A$ of weighted order $J$ forms a $\left(d_{0}, d_{1}, \ldots, d_{k}\right)$-ideal of $R$. Hence, we have $q \in A$ of weighted order $J$ with leading coefficient 1 . Using Lemma 16 again, all other coefficients of $q$ are $\left(d_{1}, \ldots, d_{k}\right)$-constants. Also, $q r-r q=0$ for any $r \in R$. 
If $q=\sum r_{L} D^{L}$, let $I$ denote the highest weighted order such that $r_{I} \notin F$ (or if all $r_{L} \in F$ let $I=e(0)$ ). For all $r \in R$, the coefficient of $D^{I}$ in $q r-r q$ is 0 . If $|J|>0$, then from the vanishing of these coefficients we get some $F$-linear combination of

$$
\left\{d_{k}^{n(k)} \ldots d_{1}^{n(1)} \mid n(i) \geq 0 \text { and } n(k)+\cdots+n(1)>0\right\}
$$

being an inner derivation by a $\left(d_{1}, \ldots, d_{k}\right)$-constant. Therefore $|J|=0$, whence $q=1$ and so $A=S$. Thus $S$ is simple.

As an example to satisfy the hypotheses of Theorem 17 , let $K$ be a field of characteristic 2 and let $x_{1}, x_{2}, \ldots$ be independent indeterminates over $K$. By Lemma 13, there exists a unique $K$-linear S.h.d. $d_{0}, d_{1}, d_{2}$ on $K\left[\left\{x_{i}\right\}\right]$ such that

$$
d_{1}\left(x_{i}\right)=x_{i+1} \text { and } d_{2}\left(x_{i}\right)=0
$$

for all $i$. Then Lemma 12 implies that $d_{0}, d_{1}, d_{2}$ extends uniquely to a S.h.d. on $R=K\left(\left\{x_{i}\right\}\right)$.

Note that $d_{1}\left(x_{i}^{2}\right)=0$ and $d_{2}\left(x_{i}^{2}\right)=x_{i+1}^{2}$ for all $i$. As $R$ is a field, we only have to show that the set

$$
\left\{d_{1}^{n(1)} d_{2}^{n(2)} \mid n(i) \geq 0 \text { and } n(1)+n(2)>0\right\}
$$

is linearly independent over the subring of central $\left(d_{1}, d_{2}\right)$-constants of $R$. Actually, we show this set is linearly independent over $R$.

If we have some $\alpha_{i j} \in R$ such that

$$
\sum_{i, j} \alpha_{i j} d_{1}^{j} d_{2}^{j}=0
$$

find $l$ such that $x_{l+h}$ is not involved in any of the $\alpha_{i j}$ for $h \geq 0$. Then

$$
\begin{aligned}
0 & =\sum_{i, j} \alpha_{i j} d_{1}^{j} d_{2}^{j}\left(x_{l}^{3}\right)=\sum_{i, j} \alpha_{i j} d_{1}^{i} d_{2}^{j}\left(x_{l} x_{l}^{2}\right) \\
& =\sum_{i, j} \alpha_{i j} d_{1}^{i}\left(x_{l} x_{l+j}^{2}\right)=\sum_{i, j} \alpha_{i j} x_{l+i} x_{l+j}^{2}
\end{aligned}
$$

Therefore each $\alpha_{i j}=0$ and the set

$$
\left\{d_{1}^{n(1)} d_{2}^{n(2)} \mid n(i) \geq 0 \text { and } n(1)+n(2)>0\right\}
$$

is linearly independent over $R$. Thus the ring $R\left[D_{1}, D_{2} ; d_{0}, d_{1}, d_{2}\right]$ is simple. 


\section{REFERENCES}

[1] S. Amitsur, Derivations in simple rings, Proc. London Math. Soc., (3) 7 (1957), 87-112.

[2] J. Cozzens and C. Faith, Simple Noetherian Rings, Cambridge University Press 1975.

[3] K. R. Goodearl and R. Warfield, Primitivity in differential operator rings, Math. Z., 180 (1982), 503-523.

[4] H. Hasse and F. K. Schmidt, Noch eine Begründung der Theorie der höheren Differentialquotienten (Zusatz bei der Korrektur), J. Reine Angew. Math., 177 (1937), 223-239.

[5] G. Hauger, Einfache Derivationspolynomringe, Arch. Math., 29 (1977), 491496.

[6] N. Heerema, Derivations and embeddings of a field in its power series ring, PAMS, 11 (1960), 188-194.

[7] - Derivations and Embeddings of a Field in its Power Series Ring II, Mich. Math. J., 8 (1961), 129-134.

[8] D. Jordan, Ore Extensions and Jacobson Rings, Ph.D. Thesis, Leeds, 1975.

[9] G. Renault, Algèbre Non Commutative, Gauthier-Villars 1975.

[10] O. Teichmuller, Differentialrechnung bei Charakteristik $p$, J. Reine Angew. Math., 175 (1937), 89-99.

[11] M. Voskoglou, Simple skew polynomial rings, Publ. Inst. Math. (Beograd) (N.S.), 37(51) 1985, 37-41.

Received September 4, 1986.

THE UNIVERSITY OF UTAH

Salt Lake City, UT 84112 


\section{PACIFIC JOURNAL OF MATHEMATICS \\ EDITORS}

V. S. VARADARAJAN

(Managing Editor)

University of California

Los Angeles, CA 90024

Herbert Clemens

University of Utah

Salt Lake City, UT 84112

R. FINN

Stanford University

Stanford, CA 94305
HERMANN FLASCHKA

University of Arizona

Tucson, AZ 85721

RAMESH A. GANGOLLI

University of Washington Seattle, WA 98195

VAUGHAN F. R. JONES

University of California

Berkeley, CA 94720
ROBION KIRBY

University of California

Berkeley, CA 94720

C. C. MOORE

University of California

Berkeley, CA 94720

HAROLD STARK

University of California, San Diego

La Jolla, CA 92093

\section{ASSOCIATE EDITORS}
R. ARENS
E. F. BECKENBACH
B. H. NEUMANN
F. WOLF
K. YOSHIDA
(1906-1982)

\section{SUPPORTING INSTITUTIONS}

UNIVERSITY OF ARIZONA

UNIVERSITY OF BRITISH COLUMBIA

CALIFORNIA INSTITUTE OF TECHNOLOGY

UNIVERSITY OF CALIFORNIA

MONTANA STATE UNIVERSITY

UNIVERSITY OF NEVADA, RENO

NEW MEXICO STATE UNIVERSITY

OREGON STATE UNIVERSITY
UNIVERSITY OF OREGON UNIVERSITY OF SOUTHERN CALIFORNIA

STANFORD UNIVERSITY

UNIVERSITY OF HAWAII

UNIVERSITY OF TOKYO

UNIVERSITY OF UTAH

WASHINGTON STATE UNIVERSITY

UNIVERSITY OF WASHINGTON 


\section{Pacific Journal of Mathematics \\ Vol. 132, No. $1 \quad$ January, 1988}

Scott W. Brown, Full analytic subspaces for contractions with rich spectrum ...1

Robert Main Burton, Jr. and Tae-Sung Kim, An invariance principle for

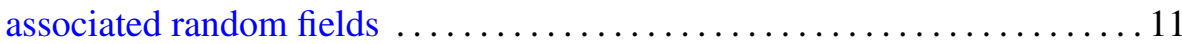

Ana M. Viola-Prioli and Jorge Viola-Prioli, Rings whose kernel functors

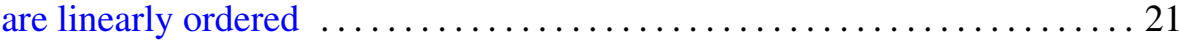

David E. Handelman, Representing polynomials by positive linear

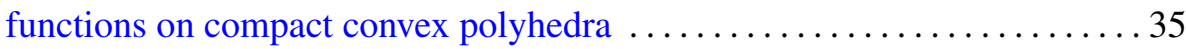

Patrick Keef, On the Tor functor and some classes of abelian groups . .....66

Dennis R. Malm, Simplicity of partial and Schmidt differential operator

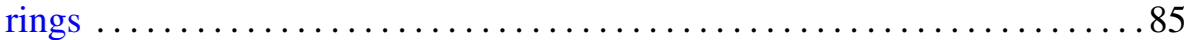

José M. Montesinos and Carmen Safont, On the Birman invariants of

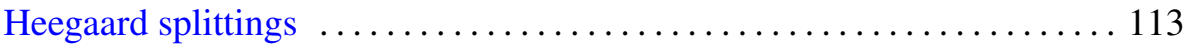

N. P. Mukherjee and Prabir Bhattacharya, The normal index of a finite group

Mario Petrich and Norman R. Reilly, Semigroups generated by certain operators on varieties of completely regular semigroups

Robert Tijdeman and Lian Xiang Wang, Sums of products of powers of given prime numbers

Joel Larry Weiner, First integrals for a direction field on a simply connected plane domain 